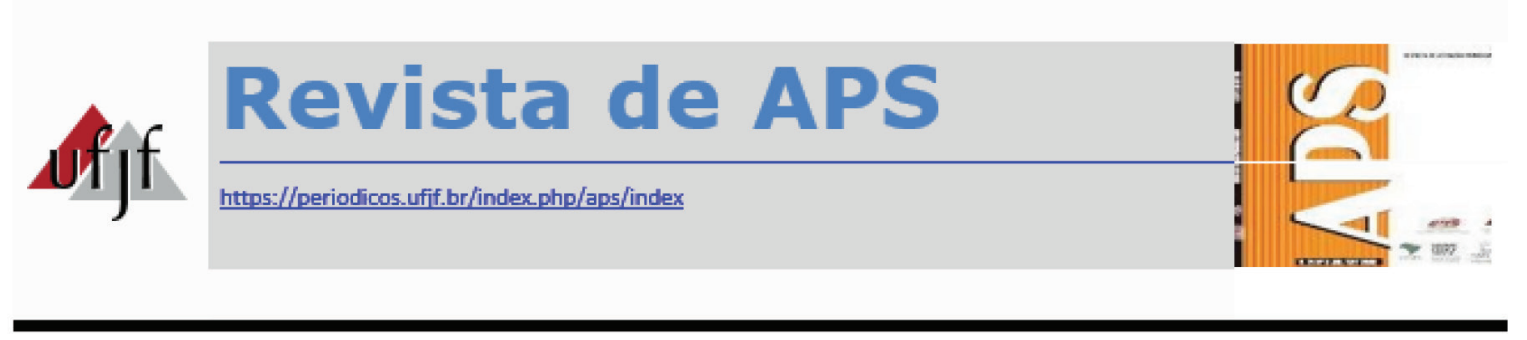

\title{
Avaliação do programa de puericultura na Atenção Primária à Saúde
}

\author{
Evaluation of the childcare program in Primary Health Care
}

\author{
Luiz Carlos Ferreira do Nascimento ${ }^{1}$, Tainara Lôrena dos Santos Ferreira², Daísy \\ Vieira de Araújo ${ }^{3}$, Fábia Barbosa de Andrade ${ }^{4}$
}

\begin{abstract}
RESUMO
Este estudo objetiva avaliar indicadores de Puericultura na Atenção Primária à Saúde na rede básica. Trata-se de um estudo quantitativo realizado com pais/responsáveis de crianças menores de dois anos atendidas em Unidades Básicas de Saúde na cidade de Santa Cruz, estado do Rio Grande do Norte, com uma amostra composta por 186 indivíduos. Este estudo vem fortalecer a proposta do Ministério da Saúde de vigilância em saúde da criança, pois se trata de uma diretriz operacional que visa à redução dos indicadores de morbimortalidade infantil no país e colabora para o fortalecimento do Pacto pela Saúde.
\end{abstract}

PALAVRAS-CHAVE: Cuidado da Criança. Criança. Atenção Primária à Saúde.

\section{ABSTRACT}

This study aims to evaluate indicators of Childcare in Primary Health Care in the basic health network. This is a quantitative study involving parents/guardians of children under two years old who are assisted in Basic Health Units in the city of Santa Cruz, State of Rio Grande do Norte, with a sample of 186 individuals. This study strengthens the proposal of the Ministry of Health for surveillance in child health, because it is an operational guideline that aims to reduce the indicators of

\footnotetext{
${ }^{1}$ Enfermeiro, administrador.

${ }^{2}$ Universidade Federal do Rio Grande do Norte - Faculdade de Ciências da Saúde do Trairi. Enfermeira. Mestra em saúde coletiva. Enfermeira plantonista da unidade mista de Jaçanã - Hospital Nossa Senhora de Fátima e do Hospital Regional Aluízio Bezerra.

${ }^{3}$ Universidade Federal do Rio Grande do Norte - Faculdade de Ciências da Saúde do Trairi. Enfermeira. Doutora em Ciências da Saúde. Professora do curso de graduação em Enfermagem da UFRN/FACISA.

${ }^{4}$ Universidade Federal do Rio Grande do Norte - Faculdade de Ciências da Saúde do Trairi. Enfermeira. Doutora em Ciências da Saúde. Professora do curso de graduação em Enfermagem da UFRN.
} 
child mortality in the country and that works to strengthen the Pact for Health.

KEYWORDS: Quality of life. Infant premature. Infant, very low birth weight. Infant, extremely low birth weight.

\section{INTRODUÇÃO}

A saúde consta na Constituição Federal Brasileira como sendo um direito de todos e um dever do Estado ${ }^{1}$. Para tanto, foi criado o Sistema Único de Saúde (SUS), a partir do projeto de lei 8080/90, que tinha por objetivo tornar esse sistema responsável por uma melhor organização das redes assistenciais do país². Como área de amplo destaque entre as ações do Ministério da Saúde emerge a área da saúde infantil, através da implementação de programas a partir do século XX até os dias atuais, quando se tem proposto um atendimento de melhor qualidade para as crianças, com o intuito de garantir um crescimento e desenvolvimento saudável e, consequentemente, diminuir a suscetibilidade às doenças e proporcionar uma melhor qualidade de vida ${ }^{3}$.

Um desenvolvimento infantil satisfatório, principalmente nos primeiros anos de vida, contribui para a formação de um sujeito com potencialidades bem desenvolvidas e com maior possibilidade de tornar-se um cidadão mais resolvido, apto a enfrentar as adversidades que a vida oferece e reduzir, assim, as disparidades sociais e econômicas da nossa sociedade ${ }^{4}$.

A assistência à saúde da criança é uma atividade de fundamental importância em função da vulnerabilidade do ser humano nessa fase do ciclo de vida. Por meio do acompanhamento da criança, papel da puericultura, a partir de aspectos de prevenção e de promoção da saúde espera-se reduzir a incidência de doenças, aumentar suas chances de crescer e desenvolverse, para alcançar todo seu potencial ${ }^{5,6}$.

No que diz respeito à rede assistencial à Saúde Infantil, a principal ferramenta utilizada pela Atenção Primária a Saúde (APS) é a consulta de puericultura, que é definida como um conjunto de ensinamentos e práticas que visam ao adequado desenvolvimento físico, psíquico e social da criança, de atuação multiprofissional, a partir de ações pautadas na promoção, prevenção e reabilitação da saúde das crianças, suas famílias e da comunidade, como um acompanhamento integral da criança, transcendendo os cuidados meramente procedimentais e curativos ${ }^{7}$.

A Atenção Básica caracteriza-se por um conjunto de ações de saúde, no âmbito individual e coletivo, que abrange a promoção e a proteção da saúde, a prevenção de agravos, o diagnóstico, o tratamento, a reabilitação e a manutenção da saúde ${ }^{8}$. Ao prover o cuidado de primeiro contato, contínuo, com ênfase em atividades de promoção e prevenção, além de forte componente de orientação familiar, os serviços efetivos de APS possibilitam todas as condições para um acompanhamento de qualidade da saúde da população infantil adscrita a eles ${ }^{9}$. 
Desta forma, por entender a importância do acompanhamento da criança como área prioritária do cuidado, o referido estudo objetivou avaliar indicadores de Puericultura na Atenção Primária à Saúde da cidade de Santa Cruz/RN, Brasil, de modo a avaliar a qualidade da assistência oferecida e apontar as suas fragilidades, haja vista que os estudos em avaliação tornam-se relevantes por serem capazes de possibilitar uma intervenção efetiva diante das dificuldades que venham a ser percebidas.

\section{METODOLOGIA}

Trata-se de um estudo epidemiológico, transversal, de caráter avaliativo, realizado com pais/responsáveis por crianças menores de dois anos, atendidas em Unidades Básicas de Saúde na cidade de Santa Cruz, estado do Rio Grande do Norte. Na zona urbana do Município de Santa Cruz existem 6 (seis) Unidades Básicas de Saúde (UBS) que funcionam de segunda a sexta e, dentre muitas atividades assistenciais, atendem, semanalmente, crianças desde seu nascimento.

O presente estudo trata de um recorte do estudo intitulado Construção $e$ validação de escala nos serviços de Puericultura: um estudo de avaliação na Atenção Primária à Saúde. A população do estudo corresponde a 600 crianças. A escolha da amostra se deu por amostragem sistemática, com base no cadastro das UBS e com respeito à proporcionalidade dos usuários cadastrados. Foi adotado ainda erro permitido de $5 \%$ e grau de confiança de $95 \%$, além de um poder de $80 \%$, mediante assinatura das mães de crianças, que resultou em uma amostra de 186 crianças, acrescido já às perdas do estudo.

Para coleta de dados foi utilizado o instrumento elaborado e validado pelo Ministério da Saúde intitulado Primary Care Assessment Tools (PCA Tools), versão para crianças, conforme os pilares da APS, entendida em elementos estruturantes, ou seja, atributos do sistema de serviços de saúde, que são: acesso de primeiro contato, integralidade, longitudinalidade, coordenação, orientação familiar e comunitária e competência cultural ${ }^{10}$.

As entrevistas foram executadas por entrevistadores alunos do curso de graduação em Enfermagem da Universidade Federal do Rio Grande do Norte (UFRN) / Faculdade de Ciências da Saúde do Trairi (FACISA), regularmente matriculados, previamente treinados após o contato realizado junto à Secretaria Municipal de Saúde de Santa Cruz/RN e, logo em seguida, nas UBS. A escolha dos sujeitos se deu de modo aleatório no momento das consultas de Puericultura, que ocorrem semanalmente nas Unidades de Saúde de Família de Santa Cruz-RN, estando essa escolha em consonância com os critérios de inclusão e exclusão. A entrevista se deu em ambiente reservado e de modo individual, após explicação dos objetivos da pesquisa pelos próprios pesquisadores, bem como 
leitura e assinatura do Termo de Consentimento Livre e Esclarecido (TCLE) por parte dos pais/responsáveis.

Os dados coletados foram armazenados no software Stata 9.0 e foram realizadas análises descritivas (frequências absoluto-relativas, média, mediana, desvio padrão) e teste qui-quadrado, considerando um Intervalo de Confiança (IC) de 95\%. O estudo foi realizado após apreciação do Comitê de Ética em Pesquisa (CEP) da Universidade Federal do Rio Grande do Norte/ Faculdade de Ciências da Saúde do Trairi, segundo o que preconiza a Resolução 466/2012 do Conselho Nacional de Saúde (CNS) sobre a pesquisa com seres humanos, sob número de parecer 348.896 .

\section{RESULTADOS}

Este estudo buscou avaliar indicadores do serviço de puericultura oferecido na Atenção Primária à Saúde do município de Santa Cruz/RN, Brasil. Para tanto, foram entrevistados 186 pais/responsáveis de crianças com idade inferior a dois anos, das quais $57 \%(n=106)$ crianças eram do sexo masculino e $43 \%(n=80)$ eram do sexo feminino. A média de idade em meses foi de 8,21; a mediana, de 7,00, e o desvio padrão, de 6,31, logo lactentes, conforme preconiza o Ministério da Saúde para consulta de puericultura. Quanto à escolaridade da mãe, em anos estudados, obteve-se média de 10,46 e mediana 9,00.

Os dados revelaram um alfa de Cronbach de 0,71 , o que mostra a existência de validade interna e dados fidedignos no constructo. Foi calculado ainda o teste de Anova Cochran, que foi significativo com $p=0,00$ e revelou também a homogeneidade das variâncias. Isso mostra que a escala de avaliação se encontra viável e fidedigna para ser usada na rede da APS, como meio de avaliar a qualidade da atenção prestada à população infantil.

No que diz respeito à facilidade em marcar a consulta de rotina da criança, observa-se que a maioria, $83,3 \%(n=155)$, afirmou com certeza ter facilidade, seguida por 8,6\% $(n=16)$ que afirmaram com certeza não. Quanto ao profissional de saúde, $84,4 \%(n=157)$ afirmaram sempre ser atendidos pelo mesmo profissional; seguidos por $7,0 \%(n=13)$ que apontaram com certeza não, enquanto $5,4 \%(n=10)$ verbalizam provavelmente sim. 
Tabela 1 - Correlação entre a facilidade em marcar consulta de revisão da criança e a procura por seu serviço de saúde para consulta de rotina antes de procurar outro serviço e a mudança do serviço de saúde se fácil de fazer. Santa Cruz/RN, Brasil, 2014

\begin{tabular}{cccccc}
\hline & $\begin{array}{c}\text { Com } \\
\text { certeza, } \\
\text { não }\end{array}$ & $\begin{array}{c}\text { Provavelmente, } \\
\text { não }\end{array}$ & $\begin{array}{c}\text { Provavelmente, } \\
\text { sim }\end{array}$ & $\begin{array}{c}\text { Com } \\
\text { certeza, } \\
\text { sim }\end{array}$ & $p<0,05$ \\
\hline $\begin{array}{c}\text { Procura pelo seu } \\
\text { serviço de saúde }\end{array}$ & $\begin{array}{c}8,6 \% \\
(n=16)\end{array}$ & $\begin{array}{c}2,2 \% \\
(n=04)\end{array}$ & $\begin{array}{c}4,3 \% \\
(n=08)\end{array}$ & $\begin{array}{c}83,3 \% \\
(n=155)\end{array}$ & 0,014 \\
$\begin{array}{c}\text { antes de procurar } \\
\text { outro serviço* }\end{array}$ & & $2,2 \%$ & $2,7 \%$ & $28,0 \%$ & 0,019 \\
$\begin{array}{c}\text { Mudança de serviço } \\
\text { de saúde se fácil de } \\
\text { fazer* }\end{array}$ & $\begin{array}{c}66,7 \% \\
(n=124)\end{array}$ & $(n=04)$ & $(n=05)$ & $(n=52)$ & \\
\hline
\end{tabular}

* Obtiveram-se valores diferentes da amostra por perdas nas questões específicas

Fonte: elaborado pelos autores

A tabela 1 se refere aos dados sobre a procura por seu serviço de saúde antes de procurar outro serviço, nos quais se verificou que $83,3 \%(n=155)$ afirmam com certeza sim. Quanto à mudança de serviço de saúde ser fácil de fazer, 66,7\% (n=124) asseguraram com certeza não mudar.

Observou-se ainda a correlação significativa entre a facilidade de marcar consultas de rotina e as variáveis acima citadas valor de $p<0,05$.

Tabela 2 - Correlação entre ser atendido sempre pelo mesmo profissional de saúde e ser compreendido pelo profissional de saúde, ter tempo suficiente durante a consulta e o profissional conhecer a história clínica da criança. Santa Cruz/RN, Brasil, 2014

\begin{tabular}{|c|c|c|c|c|c|}
\hline & $\begin{array}{c}\text { Com } \\
\text { certeza, } \\
\text { não }\end{array}$ & $\begin{array}{l}\text { Provavelmente, } \\
\text { não }\end{array}$ & $\begin{array}{l}\text { Provavelmente, } \\
\text { sim }\end{array}$ & $\begin{array}{l}\text { Com } \\
\text { certeza, } \\
\text { sim }\end{array}$ & $p<0,05$ \\
\hline $\begin{array}{l}\text { Profissional } \\
\text { de saúde } \\
\text { compreender o } \\
\text { usuário* }\end{array}$ & $\begin{array}{l}2,2 \% \\
(n=04)\end{array}$ & $\begin{array}{l}2,2 \% \\
(n=04)\end{array}$ & $\begin{array}{c}8,6 \% \\
(n=16)\end{array}$ & $\begin{array}{c}86,6 \% \\
(n=161)\end{array}$ & 0,004 \\
\hline $\begin{array}{l}\text { Tempo suficiente } \\
\text { para falar sobre } \\
\text { suas preocupações }\end{array}$ & $\begin{array}{l}1,1 \% \\
(n=02)\end{array}$ & $\begin{array}{l}1,1 \% \\
(n=02)\end{array}$ & $\begin{array}{l}1,6 \% \\
(n=03)\end{array}$ & $\begin{array}{c}96,2 \% \\
(n=179)\end{array}$ & 0,006 \\
\hline $\begin{array}{l}\text { Profissional } \\
\text { de saúde ter } \\
\text { conhecimento } \\
\text { da história clínica } \\
\text { (médica) da } \\
\text { criança* }\end{array}$ & $\begin{array}{l}8,1 \% \\
(n=15)\end{array}$ & $\begin{array}{c}3,8 \% \\
(n=07)\end{array}$ & $\begin{array}{c}4,8 \% \\
(n=09)\end{array}$ & $\begin{array}{c}81,7 \% \\
(n=152)\end{array}$ & 0,000 \\
\hline
\end{tabular}

* Obtiveram-se valores diferentes da amostra por perdas nas questões específicas

Fonte: elaborada pelos autores 
$\mathrm{Na}$ tabela 2, pode-se observar uma correlação significativa entre ser atendido pelo mesmo profissional de saúde e as variáveis: Profissional de saúde compreender o usuário, na qual se percebeu que $86,6 \%$ ( $n=161$ ) acreditam que o profissional de saúde entende o que ela(e) pergunta sobre a saúde de sua criança; Ter tempo suficiente para falar sobre seus problemas e preocupações, a partir da qual se verificou que $96,2 \%(n=179)$ dos sujeitos consideram o tempo da consulta suficiente. $E$, quanto ao profissional conhecer a história clínica completa de sua criança, 81,7\% ( $n=152$ ) afirmam com certeza sim.

\section{DISCUSSÃO}

Sabe-se que a criança mostra-se como prioridade e se constitui no grupo mais vulnerável da humanidade, em busca da garantia de um crescimento e desenvolvimento saudável ${ }^{3}$. Assim, apresentam-se como fundamentais os estudos de avaliação que visam verificar se as consultas de saúde da criança estão atendendo, ou não, as necessidades e perspectivas da população. Dessa forma, este estudo buscou avaliar os indicadores de Puericultura na Atenção Primária.

No que concerne à facilidade em marcar a consulta de rotina da criança, observase que a acessibilidade ao serviço tem sido proposta, o que contribui para a efetividade da assistência e facilita o acompanhamento da criança na APS. O atendimento pelo mesmo profissional de saúde, como afirma a maioria da amostra, propõe o fortalecimento da tríade usuário-profissional-serviço e coopera para uma assistência em saúde norteada pela realidade dos usuários, que também é proposta da Atenção Básica, por ser um nível de assistência centrado na comunidade, com fins de promoção e proteção da saúde.

A tabela 1, referente aos dados de utilização do serviço e de acessibilidade, demonstra que o serviço de saúde da própria comunidade tem sido procurado antes de procurar outro serviço, fato atribuído à facilidade em marcar a consulta de revisão. A satisfação do usuário também é aqui avaliada através da confirmação "com certeza não", feita pela maioria da amostra quando questionada quanto a pretender mudança de serviço de saúde, o que aponta, na ótica do usuário, que a assistência prestada tem sido satisfatória.

O acesso, como a possibilidade da consecução do cuidado de acordo com as necessidades, tem inter-relação com a resolubilidade e extrapola a dimensão geográfica, tendo em vista que abrange aspectos de ordem econômica, cultural e funcional de oferta de serviços ${ }^{11}$. Para que exista o acesso aos serviços de saúde, é necessário que existam serviços em quantidade e qualidade, que disponham de profissionais qualificados, equipamentos adequados, localização acessível à comunidade e que abranja as necessidades da população local, de modo que garanta a qualidade do serviço prestado e a satisfação da clientela ${ }^{12}$. 
A estratégia de marcação de consultas interfere diretamente na busca pelo serviço para realização das mesmas, assim como na satisfação das mães. Dessa forma, estratégias em que se utiliza a marcação de consulta feita pelos Agentes Comunitários de Saúde (ACS), ou a marcação feita logo após a consulta, promove a continuidade da assistência através da facilidade em marcar a consulta de revisão.

Na tabela 2, quanto ao profissional de saúde compreender o usuário e, durante a consulta, o usuário ter tempo suficiente para falar os problemas e preocupações, observase a positividade de tais ações na APS, uma vez que reflete na relação interpessoal e possibilita um ambiente de confiança entre a população e os profissionais do serviço de saúde, favorecendo a continuidade da atenção à saúde da criança.

Aintegralidade, quando centrada no sujeito (em suas necessidades e expectativas), o objeto da atenção dos serviços e de seus profissionais, leva as ações de saúde à discussão de questões transversais como a efetividade, continuidade e terminalidade do cuidado ofertado ${ }^{13}$. Vale destacar que o paciente que busca os serviços de saúde traz consigo, além da queixa, suas necessidades, desejos, dificuldades, enfim, todas suas demandas. Logo, se quiser cuidá-lo, tem que saber perceber, escutar, acolher e decifrar essa demanda, ou seja, suas necessidades de saúde.

Ainda na tabela 2, quanto ao profissional de saúde ter conhecimento da história clínica (médica) da criança, percebe-se a positividade da assistência prestada na rede da APS, seja pelo seu caráter comunitário e familiar, considerando o usuário em um contexto biopsicossocial, seja por sempre ser atendido pelo mesmo profissional, como também foi observado em nosso estudo.

O cenário do cuidado na rede da APS e a realização das consultas de puericultura nas Unidades Básicas de Saúde (UBS) são instituídos com vistas a garantir o grande atributo da APS, a longitudinalidade e garantia de continuidade/seguimento das mães/ responsáveis que realizam as consultas mensais de seus filhos, quando buscam a identificação precoce de doenças, bem como a evolução da criança nos aspectos físicos, sociais e psíquicos, em relação ao ambiente onde ela se encontra inserida.

A longitudinalidade do cuidado em saúde tem grande relevância no acompanhamento do crescimento e desenvolvimento da criança, com especial atenção nos primeiros anos de vida, tendo em vista que seu principal objetivo é a saúde física, emocional e a competência social para o aprendizado ${ }^{14,15}$.

A necessidade de atenção à saúde da criança deve ser uma prioridade nas comunidades locais. Esse cuidado tem a missão de tratar a saúde das crianças em uma área definida, além de revelar oportunidades que foram apresentadas em tempo real, com a participação da escola e a educação para seus alunos e suas famílias, embora considerando um conjunto de características politicamente difíceis ${ }^{16}$.

Portanto, no que diz respeito à longitudinalidade nos atendimentos em puericultura, percebe-se que as Unidades Básicas de Saúde da Cidade de Santa Cruz/ 
RN atendem às necessidades da população. Não é possível listar nenhuma fragilidade que comprometa a prestação de cuidados longitudinais. Ao contrário, observa-se que há uma relação de afinidade e confiança por parte dos usuários em relação ao seu serviço de saúde, fato que fortalece ainda mais o cuidado à criança, além de prevenir e promover, com qualidade, os agravos à saúde da criança.

\section{CONCLUSÃO}

Os resultados da pesquisa revelaram a positividade do serviço, pois tem sido acessível à população e realizado pelo mesmo profissional, que na ótica de usuários conhece também a história clínica completa da criança. Por ser considerada porta de entrada para os demais níveis de atenção, a APS ainda contribui para a satisfação do usuário através de se sentir ouvido e compreendido e possuir tempo suficiente para a consulta, o que fortalece também a continuidade da assistência e as linhas de cuidado.

Assim, a avaliação de serviços de saúde vai ao encontro do que é proposto pelo Ministério da Saúde através do Programa de Melhoria da Qualidade da Atenção na rede da APS, uma vez que busca a qualidade da atenção à saúde e a efetividade da assistência das ações e serviços prestados à população, bem como fortalece a proposta de vigilância em saúde da criança, pois se trata de uma diretriz operacional que visa à redução dos indicadores de morbimortalidade infantil no país e colabora para o fortalecimento do Pacto pela Saúde.

O monitoramento de ações e resultados e a avaliação continuada de serviços de saúde proporcionam conhecer as reais necessidades do serviço, a qualidade da assistência prestada, a satisfação de usuários e são fundamentais para adequação de políticas públicas de saúde e implementação de ações. Espera-se, assim, que este estudo venha impulsionar outras realidades de municípios brasileiros na avaliação e direcionamento de estratégias a fim de um alcance de maiores indicadores previstos na política de Atenção Primária à Saúde.

\section{REFERÊNCIAS}

1. Brasil. Constituição (1988). Constituição da República Federativa do Brasil. Brasília, DF: Senado Federal: Centro Gráfico; 1988. 292 p.

2. Brasil. Lei no 8.080, de 19 de setembro de 1990. Lei Orgânica da Saúde. Dispõe sobre as condições para a promoção, proteção e recuperação da saúde, a organização e o funcionamento dos serviços correspondentes e dá outras providências. Brasília, set. 1990.

3. Brasil. Ministério da Saúde. Secretaria de Atenção à Saúde. Departamento de Ações Programáticas Estratégicas. Política Nacional de Atenção Integral à Saúde da Criança: 
orientações para implementação / Ministério da Saúde. Secretaria de Atenção à Saúde. Departamento de Ações Programáticas Estratégicas. Brasília: Ministério da Saúde, 2018.

4. Organização Panamericana de Saúde (OPAS). Manual para vigilância do desenvolvimento infantil no contexto da AIDPI. Washington, D.C.: OPAS; 2005.

5. Campos RMC, Ribeiro CA, Silva CV, et al. Consulta de enfermagem em puericultura: a vivência do enfermeiro na Estratégia de Saúde da Família. Rev. esc. enferm. USP [online]. 2011; 45(3):566-74.

6. Del Ciampo LA. et al. O programa de saúde da família e a puericultura. Ciência \& Saúde Coletiva. 2006; 11(3):739-43.

7. Góes FGB, Silva MA, Paula GK, Oliveira LPM, Mello NC, Silveira SSD. Contribuições do enfermeiro para boas práticas na puericultura: revisão integrativa da literatura. Rev. Bras. Enferm. 2018; 71( Suppl 6 ):2808-17. Disponível em: http://www.scielo. $\mathrm{br} /$ scielo.php?script=sci_arttext\&pid=S0034-71672018001202808\&lng=pt. http:// dx.doi.org/10.1590/0034-7167-2018-0416.

8. Brasil. Ministério da Saúde. Gabinete do Ministro. Portaria no 2.488, de 21 de outubro de 2011. Aprova a Política Nacional de Atenção Básica, estabelecendo a revisão de diretrizes e normas para a organização da Atenção Básica, para a Estratégia Saúde da Família - ESF e o Programa de Agentes Comunitários de Saúde - PACS. Diário Oficial da República Federativa do Brasil, Brasília, DF, 24 out. 2011. Disponível em: http://bvsms.saude.gov.br/bvs/saudelegis/gm/2011/9. prt2488_21_10_2011.html.

9. Harzheim E, Stein AT, Álvarez-Dardet C. A efetividade dos atributos na atenção primária sobre a saúde infantil. Rev. Boletim de saúde, Porto Alegre, 2004; 18(1): 23-39.

10. Starfield B. Atenção primária: equilíbrio entre necessidades de saúde, serviços e tecnologia / Bárbara Starfield. Brasília: UNESCO, Ministério da Saúde, 2002; 726p.

11. Souza ECF, et al. Acesso e acolhimento na atenção básica: uma análise da percepção dos usuários e profissionais de saúde. Cad. Saúde Pública [online]. 2008; 24(suppl.1): s100-s110.

12. Marsiglia RMG. Universalização do acesso ao Sistema Único de Saúde no Brasil: desafios para a Atenção Primária à Saúde. Cad. Ter. Ocup. UFSCar, São Carlos. 2012; 20(3):317-25.

13. Favoreto CAO. A prática clínica e o desenvolvimento do cuidado integral à saúde no contexto da Atenção Primária. Rev. APS. jan/mar 2008; 11(1):100-08. Disponível em: http://www.ufff.br/nates/files/2009/12/100-108.pdf.

14. Baratieri T, Marcon SS. Longitudinalidade do cuidado: compreensão dos enfermeiros que atuam na estratégia saúde da família. Esc Anna Nery. 2011; 15(4):802-810. 
15. Mello DF, et al. Seguimento da saúde da criança e a longitudinalidade do cuidado. Rev Bras Enferm, Brasília. 2012; 65(4):675-9.

16. Lewin LO, Giudice E, Czinn SJ. Let's Start at the Very Beginning: Addressing the Goal of Service to the Community. The Journal of Pediatrics. 2014; 164(3):434-5.

Submissão: janeiro de 2015.

Aprovação: maio de 2019. 